\title{
Conhecimento e atitudes da equipe de enfermagem sobre cateter com dispositivo seguro em um hospital brasileiro
}

\author{
Nursing staff knowledge and attitudes about safe device catheters in a Brazilian hospital \\ Conocimientos y actitudes del personal de enfermería sobre catéteres de dispositivos seguros en un \\ hospital brasileño
}

\begin{abstract}
Priscila do Carmo Freitas de CarvalhoI, Gabriela da Cunha Januário ${ }^{I I}$, Damiana Aparecida Trindade Monteiro ${ }^{\mathrm{III}}$, Silmara Elaine Malaguti Toffano ${ }^{\mathrm{IV}}$
\end{abstract}

Resumo: Objetivo: descrever o conhecimento e atitudes dos profissionais de enfermagem sobre um Dispositivo Seguro com Controle de Engenharia. Método: estudo transversal descritivo, realizado em um hospital público brasileiro, especializado para o tratamento de HIV/aids. Foram entrevistados profissionais de enfermagem no período de maio a julho de 2015. Os dados foram analisados por meio da estatística descritiva e inferencial. Resultados: dos 180 entrevistados, $72,2 \%$ não participaram de treinamento que propiciava conhecimento para o uso do cateter com dispositivo de segurança. Quanto às atitudes de risco, a reinserção de um mesmo cateter periférico antes de acionar a proteção da agulha, em mais de uma punção, no mesmo paciente foi citada como prática rotineira por $48(26,7 \%)$ participantes. Conclusão: a maioria dos profissionais de enfermagem não recebeu treinamentos para uso de dispositivos, mas reconheceu características e mecanismos de proteção contra picada de agulha e contato com sangue.

Descritores: Profissionais de enfermagem; Ferimentos penetrantes produzidos por agulha; Cateteres; Saúde do trabalhador

\begin{abstract}
Objective: describing the knowledge and attitudes of nursing professionals regarding a Safe Device with Engineered Controls. Methods: cross-sectional descriptive study, carried out in a Brazilian general hospital, specialized in the treatment of HIV/aids. Nursing professionals were interviewed from May to July 2015. Data was analyzed using descriptive and inferential statistics. Results: from the 180 interviewees, $72.2 \%$ did not participate in any training that offered knowledge about the use of the catheter with a safety device. Regarding the risk attitudes, reinserting the same peripheral catheter before activating the needle protection, in more than one puncture, in the
\end{abstract}

\footnotetext{
I Enfermeira. Mestre. Servidora da Fundação Hospitalar do Estado de Minas Gerais (FHEMIG). Belo Horizonte, MG, Brasil. E-mail: prisciladcf@yahoo.com.br; ORCID: https://orcid.org/0000-0003-3933-4510

II Enfermeira. Mestre. Docente na Universidade Estadual de Minas Gerais. Passos, MG. Brasil. E-mail: gabriela_cunha92@hotmail.com; ORCID: https://orcid.org/0000-0002-3585-0705

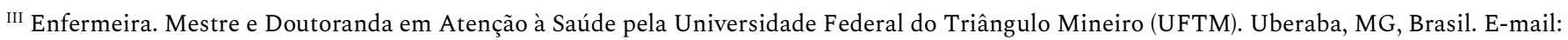
damianaatm@hotmail.com; ORCID: https://orcid.org/0000-0002-6740-7687

IV Enfermeira. Doutora em Ciências da Saúde. Docente da Universidade Federal do Triangulo Mineiro. Uberaba, MG, Brasil. E-mail: silmara.toffano@uftm.edu.br; ORCID: https:/orcid.org/0000-0002-9080-9123
} 
Conhecimento e atitudes da equipe de enfermagem sobre cateter com dispositivo seguro... $\mid 2$

same patient, was mentioned as a routine practice by $48(26.7 \%)$ participants. Conclusion: most nursing professionals did not receive training in the use of the devices but could recognize characteristics and mechanisms of protection against needle punctures and contact with blood.

Descriptors: Nurse Practitioners; Needlestick Injuries; Catheters; Occupational Health

Resumen: Objetivo: describir el conocimiento y las actitudes de los profesionales de enfermería sobre un Dispositivo de Seguridad con Controlo de Ingeniería. Método: estudio trasversal descriptivo, conducido en un hospital público brasileño, especializado en el tratamiento de HIV/sida. Se entrevistó profesionales de enfermería de mayo a julio de 2015. Se analizó a los datos utilizando estadística descriptiva e inferencial. Resultados: $72.2 \%$ de los 180 entrevistados no participaron de ningún entrenamiento sobre el uso del catéter con dispositivo de seguridad. Con respecto a las actitudes de riesgo, la reinserción del mismo catéter periférico antes de accionar la protección de la aguja, en más de una punción, en el mismo paciente, fue citada como práctica común por 48 (26.7\%) de los participantes. Conclusión: la mayoría de los profesionales de enfermería no recibió entrenamiento para utilizar los dispositivos, pero reconoció características y mecanismos de protección contra pinchazos y contacto con la sangre.

Descriptores: Enfermeras Practicantes; Lesiones por Pinchazo de Aguja; Catéteres; Salud Laboral

\section{Introdução}

A enfermagem é a categoria profissional mais encontrada nas instituições hospitalares, e em sua rotina realiza procedimentos diversos com uma frequência maior no cuidado direto ao paciente, sendo a principal executora da punção venosa periférica (PVP). Dessa forma, como consequência, há uma constante manipulação e descarte de materiais perfurocortantes, o que favorece a exposição a material biológico e ao risco de acidentes. Dentre os riscos em que o profissional da enfermagem está exposto, destaca-se o risco biológico, caracterizado como a exposição a agentes biológicos como microrganismos, modificados ou não geneticamente, as culturas de células, os parasitas, as toxinas e os príons. Para a proteção desses profissionais, são necessários conhecimento e atenção voltados para a realização desses procedimentos. ${ }^{1-3}$

Devido a essa rotina, a exposição ocupacional a material biológico durante a PVP tem sido documentada na literatura, envolvendo principalmente profissionais de enfermagem. ${ }^{4}$ Dados da literatura mostraram que grande parte dos acidentes de trabalho envolvendo profissionais de enfermagem ocorreu com materiais perfurocortantes $77,7 \% .^{5}$ 
Conhecimento e atitudes da equipe de enfermagem sobre cateter com dispositivo seguro... $\mid 2$

Considerando os fatores de risco inerentes as atividades realizadas pelos profissionais de enfermagem, como o uso de cateter venoso periférico, o risco de contato com sangue, a lesão percutânea, a carga horária de trabalho, o tempo de experiência e ausência de treinamentos para manuseio de perfurocortantes ${ }^{4,6}$ torna-se importante ressaltar o uso de Dispositivos Seguros com Controle de Engenharia (DSCE). ${ }^{7-9}$ Isto é fundamental para evitar não só acidente com material perfurocortante como também o contato com o sangue..$^{7-9}$

Os DSCE são materiais usados para a PVP e outros procedimentos que contam com diferentes características para proteger a ponta da agulha ou toda a agulha ou ainda, com mecanismos que impedem ou minimizam o contato com sangue antes ou após a punção. ${ }^{10-11}$ São classificados de acordo com o mecanismo de ativação de proteção da agulha, ou seja: DSCE com técnica de ativação passiva, no qual o usuário não precisa ativar nenhum recurso para a proteção da agulha após o uso e, com técnica de ativação ativa, ou seja, para que a agulha fique protegida após o uso, o usuário precisará acionar algum mecanismo de segurança, como botão, tampa ou trava. ${ }^{10}$

Com o intuito de reduzir as picadas de agulha e/ou à exposição a sangue, o uso do DSCE tornou-se obrigatório em alguns países. ${ }^{4,12}$ No Brasil em 2008, a Portaria GM nº.939, de 18 de novembro, estabeleceu o prazo de seis meses para divulgação dos dispositivos e treinamento; 18 meses após o prazo de divulgação e treinamento para implementação e adaptação de mercado e 24 meses a partir da data de publicação da portaria, para que os empregadores façam a substituição dos materiais perfurocortantes por outros com dispostivo de segurança. ${ }^{13} \mathrm{~A}$ legislação e as portarias têm estabelecido em suas diretrizes o uso desses dispositivos, a fim de reduzir acidentes com materiais perfurocortantes. ${ }^{1,13}$

Estudos internacionais apontaram para redução no número de acidentes percutâneos, após a introdução dos DSCE, associados a programas de capacitação ou treinamento., ${ }^{914-15}$ No Brasil, uma investigação apontou que a adoção de um único dispositivo de segurança reduziu, significativamente, o número de acidentes percutâneos entre a equipe de enfermagem. ${ }^{8}$ 
Por se tratar de uma estratégia inovadora, de alto custo para o sistema de saúde e que contribui diretamente para os programas de prevenção de acidentes envolvendo profissionais de enfermagem, foi feita a seguinte questão de pesquisa: qual o conhecimento dos profissionais de enfermagem que trabalham em um hospital especializado em doenças infectocontagiosas acerca de um novo dispositivo para PVP? Nesse sentido, esta investigação teve como objetivo descrever o conhecimento e atitudes dos profissionais de enfermagem sobre um DSCE.

\section{Método}

Trata-se de um estudo transversal descritivo, em que a coleta de dados foi realizada no período de maio a julho de 2015, em um hospital público brasileiro, especializado para o tratamento de HIV/aids, que desde o final de 2008 adotou DSCE para PVP.

No período de coleta de dados a instituição contava com um DSCE com sistema passivo de segurança. A proteção é efetivada após a retirada da agulha da cânula e sua ativação segura independe da ação do usuário, com a cobertura da ponta da agulha.

A população foi composta inicialmente por 210 profissionais de enfermagem, distribuídos em três categorias: enfermeiros, técnicos e auxiliares de enfermagem. Considerando os critérios de inclusão (atuar na assistência direta ao paciente) e de exclusão (afastamento do trabalho durante o período de coleta de dados) 184 profissionais estavam aptos a participarem da pesquisa.

Para tanto, um instrumento com questões abertas e fechadas contemplando: variáveis sobre a caracterização sociodemográfica, profissional, ocorrência de acidentes; aspectos referentes aos DSCE usados atualmente, como facilidades e dificuldades do seu uso. Tal instrumento foi construído pelos autores e submetido à apreciação de três especialistas na temática, que procederam à avaliação quanto à forma e a pertinência das questões em relação ao objetivo da pesquisa. O estudo piloto foi realizado com 10 profissionais de enfermagem da unidade ambulatorial, quatro enfermeiros e seis técnicos de enfermagem do mesmo hospital. O 
Conhecimento e atitudes da equipe de enfermagem sobre cateter com dispositivo seguro... $\mid 4$

questionário desses profissionais não foi incluído no estudo final. O instrumento de coleta de dados foi respondido por 180 profissionais de enfermagem, houve uma perda de quatro profissonais que não foram encontrados no setor de trabalho nos dias da coleta de dados.

Os dados foram coletados pelos pesquisadores, que durante todos os turnos de trabalho, abordavam os profissionais, nos momentos mais oportunos. Após compreensão dos objetivos da pesquisa e assinatura do Termo de Consentimento Livre e Esclarecido (TCLE), os participantes respondiam ao instrumento de coleta de dados, os quais eram guardados em envelopes separados e lacrados pelos pesquisadores. Posteriormente, os envelopes eram abertos e os dados digitados em um banco de dados no Excel. A análise estatística descritiva e inferencial (testes Kolmogorov-Smirnov e Qui-Quadrado; $<<0,05)$ foi feita no software IBM SPSS ${ }^{\circledR}$, versão 22.

A pesquisa foi aprovada pelo Comitê de Ética do Hospital, sob o Parecer nª 956.314 e registro CAAE: 35510714.2.3001.5124, de 10 de fevereiro de 2015 e pelo Comitê de Ética da Universidade, sob o Parecer n 863.814 e registro CAAE: 35510714.2 .0000 .5545 , de 30 de outubro de 2014. Os autores conduziram a investigação conforme os princípios éticos das resoluções referentes às pesquisas com seres humanos.

\section{Resultados}

Dos participantes, 27 (15,0\%) eram enfermeiros, 140 (77,7\%) técnicos de enfermagem e 13 (7,2\%) auxiliares de enfermagem; a maioria $(53,9 \%)$ do sexo masculino; média de idade de 40,2 anos, com Desvio Padrão $(\mathrm{DP})=8,33$, com menos de cinco anos de experiência de trabalho ( $n=138 / 76,7 \%)$. A média de horas trabalhadas na semana (somando-se outros locais) foi de 47,8 $(\mathrm{DP}=17,2)$.

Com relação ao setor de trabalho, 62,8\% lotados em unidades de internação, 30,0\% em unidade de terapia intensiva (UTI) e 1,1\% na assistência domiciliar terapêutica.

Dos $50(27,8 \%)$ profissionais que disseram ter feito treinamento para uso de DSCE, $20,0 \%$ 
afirmaram que o mesmo foi oferecido pelo hospital e 6,7\% pelo fabricante do produto; outros dois $(1,1 \%)$ não responderam. Ao comparar a realização de treinamentos para uso do DSCE com a categoria profissional, foi evidenciada diferença estatística $(\mathrm{p}=0,009)$, ou seja, o percentual de treinamentos foi significativamente superior no grupo Enfermeiro, no que nas demais categorias.

A Tabela 1 apresenta a caracterização dos profissionais de enfermagem de acordo com a categoria ocupacional, sexo, idade, setor, tempo de trabalho e treinamento para o uso do DSCE.

Tabela 1 - Caracterização dos profissionais de enfermagem de um hospital público, segundo categoria, sexo e setor de trabalho. Belo Horizonte/MG, 2015.

\begin{tabular}{|c|c|c|c|}
\hline Variável & & $\mathrm{N}$ & $\%$ \\
\hline \multirow[t]{3}{*}{ Categoria } & Enfermeiro & 27 & 15,0 \\
\hline & Técnicos de enfermagem & 140 & 77,7 \\
\hline & Auxiliares de enfermagem & 13 & 7,2 \\
\hline \multirow[t]{2}{*}{ Sexo } & Feminino & 83 & 46,1 \\
\hline & Masculino & 97 & 53,9 \\
\hline \multirow[t]{5}{*}{ Idade } & $<19$ & 12 & 6,7 \\
\hline & $20-29$ & 13 & 7,2 \\
\hline & $30-39$ & 72 & 42,9 \\
\hline & $40-49$ & 55 & 30,6 \\
\hline & $>50$ & 28 & 15,6 \\
\hline Setor de trabalho & Internação & 113 & 62,8 \\
\hline
\end{tabular}


Conhecimento e atitudes da equipe de enfermagem sobre cateter com dispositivo seguro... 6

Unidade de Terapia Intensiva

Bloco Cirúrgico

Hospital dia

Assistência Domiciliar Terapêutica

Tempo de trabalho na $<5$

instituição

$5-10$

$>10$

Curso/Treinamento para Sim uso de dispositivo seguro

com controle de Não engenharia
54

07

04

02

138

15

27

50

130
30,0

3,9

2,2

1,1

76,7

15,0

27,8

72,2

Fonte: Dados da pesquisa.

A Tabela 2 descreve respostas quanto ao conhecimento dos profissionais de enfermagem acerca das características do DSCE usado no hospital no período da investigação.

Dos entrevistados $77,2 \%$ responderam não se sentirem protegidos da picada de agulha e contato com o sangue utilizando o DSCE atual, a maioria, 81,1\%, afirmou que houve mudança na técnica de inserção comparada com o cateter convencional. Do total de profissionais entrevistados, $60,6 \%$ disseram que o DSCE permite à cobertura da ponta da agulha e $78,3 \%$ a sua cobertura total após o uso. Em relação ao contato com sangue ao utilizar o cateter com dispositivo, $81,1 \%$ dos profissionais relataram que o dispositivo não permite contato com sangue durante a punção. Sobre o contato com sangue após o procedimento de punção, 78,3\% afirmaram que o mesmo permite contato com este fluido. 
Tabela 2 - Respostas dos participantes referentes às características de um DSCE para PVP. Belo Horizonte/MG, 2015.

\begin{tabular}{|c|c|c|c|c|c|c|}
\hline \multirow[t]{2}{*}{ Variável } & \multicolumn{2}{|c|}{ Sim } & \multicolumn{2}{|c|}{ Não } & \multicolumn{2}{|c|}{$\begin{array}{c}\text { Em } \\
\text { Branco }\end{array}$} \\
\hline & $\mathrm{N}$ & $\%$ & $\mathrm{~N}$ & $\%$ & $\mathrm{~N}$ & $\%$ \\
\hline $\begin{array}{l}\text { Sente-se protegido da picada de } \\
\text { agulha e contato com o sangue } \\
\text { utilizando o cateter com dispositivo } \\
\text { de segurança atual }\end{array}$ & 35 & 19,4 & 139 & 77,2 & 06 & 3,3 \\
\hline $\begin{array}{l}\text { Houve mudança na técnica de } \\
\text { inserção (comparada com o cateter } \\
\text { convencional) }\end{array}$ & 146 & 81,1 & 24 & 13,3 & 10 & 5,6 \\
\hline $\begin{array}{l}\text { Permite a cobertura da ponta da } \\
\text { agulha }\end{array}$ & 109 & 60,6 & 71 & 39,4 & - & - \\
\hline $\begin{array}{l}\text { Permite a cobertura total da agulha } \\
\text { após o uso }\end{array}$ & 39 & 21,7 & 141 & 78,3 & - & - \\
\hline $\begin{array}{l}\text { Permite contato com sangue durante } \\
\text { a punção }\end{array}$ & 34 & 18,9 & 146 & 81,1 & - & - \\
\hline $\begin{array}{l}\text { Permite contato com sangue após a } \\
\text { punção }\end{array}$ & 141 & 78,3 & 39 & 21,7 & - & - \\
\hline
\end{tabular}

Fonte: Dados da pesquisa.

A Tabela 3 apresenta as facilidades e as dificuldades sobre o uso de DSCE.

Tabela 3 - Facilidades e dificuldades descritas pelos profissionais de enfermagem sobre um DSCE para PVP. Belo Horizonte/MG,2015.

\begin{tabular}{lcccc}
\hline Variável & \multicolumn{2}{c}{ Sim } & \multicolumn{2}{c}{ Não } \\
\cline { 2 - 5 } & $\mathrm{N}$ & $\%$ & $\mathrm{~N}$ & $\%$ \\
\hline Facilidades & 141 & 78,3 & 39 & 21,7 \\
$\quad$ Proteção contra picada de agulha & 57 & 31,7 & 123 & 68,3 \\
Proteção contato com sangue & 92 & 51,1 & 88 & 48,9 \\
Praticidade & 81 & 45,0 & 99 & 55,0
\end{tabular}


Conhecimento e atitudes da equipe de enfermagem sobre cateter com dispositivo seguro... $\mid 8$

Nenhuma facilidade

Dificuldades

Proteção somente da ponta da agulha

Falta de treinamento

Falta de habilidade

Manuseio difícil

Nenhuma dificuldade
03

1,7 32

44

12

20

84

$\begin{array}{ccc}17,8 & 148 & 82,2 \\ 24,4 & 136 & 75,6 \\ 6,7 & 168 & 93,3 \\ 11,1 & 160 & 88,9 \\ 46,7 & 96 & 53,3\end{array}$

Fonte: Dados da pesquisa.

Quanto às facilidades descritas pelos profissionais de enfermagem com a introdução do DSCE, a mais citada foi à proteção contra picada de agulha (78,3\%). Em relação às dificuldades abordadas, a maioria dos profissionais não afirmou nenhuma dificuldade, tanto de manuseio, quanto de treinamento ou proteção somente da ponta da agulha.

Quanto à segurança percebida, $166(92,2 \%)$ responderam que o cateter com dispositivo seguro atual não funcionava de maneira confiável, pois atribuíram que o cateter usado protegia apenas a ponta da agulha e permitia o contato com o sangue e como trabalhavam em hospital de doenças infecciosas tal proteção seria fundamental; nove $(5,0 \%)$ responderam que confiavam na proteção do dispositivo e cinco $(2,8 \%)$ não souberam responder. Não houve diferença significativa $(\mathrm{p}=0,85)$ ao comparar a categoria profissional e a confiança no dispositivo usado na instituição.

Ainda nesta questão, 18 (10,0\%) responderam que já passaram por alguma situação em que o mesmo não foi acionado de maneira correta após a punção; $11(6,7 \%)$ não responderam.

A Tabela 4 apresenta as atitudes dos profissionais quanto ao uso de luvas e reinserção do cateter com DSCE. 
9 | Carvalho PCF, Januário GC, Monteiro DAT, Toffano SEM

Tabela 4 - Atitude dos profissionais de enfermagem quanto ao uso de luvas e reinserção do cateter com DSCE.

\begin{tabular}{|c|c|c|c|c|c|c|}
\hline \multirow[t]{2}{*}{ Variável } & \multicolumn{2}{|c|}{ Sim } & \multicolumn{2}{|c|}{ Não } & \multicolumn{2}{|c|}{ Às vezes } \\
\hline & $\mathrm{N}$ & $\%$ & $\mathrm{~N}$ & $\%$ & $\mathrm{~N}$ & $\%$ \\
\hline $\begin{array}{l}\text { Você utiliza luvas de procedimento em } \\
\text { todas as punções venosas com cateter } \\
\text { com DSCE usado atualmente }\end{array}$ & 167 & 92,8 & 04 & 2,2 & 09 & 5,0 \\
\hline $\begin{array}{l}\text { Você já reutilizou o cateter venoso } \\
\text { periférico com DSCE antes de acionar } \\
\text { a proteção da agulha em mais de uma } \\
\text { punção (comparada com o cateter } \\
\text { convencional) }\end{array}$ & 48 & 26,7 & 125 & 69,4 & 07 & 3,9 \\
\hline
\end{tabular}

Em relação às atitudes de risco para a segurança do profissional e do paciente, a PVP sem luvas foi citada por quatro $(2,2 \%)$, com a justificativa de atrapalhar a palpação da veia; nove $(5,0 \%)$ responderam que as utilizavam somente em algumas situações e outros $167(92,8 \%)$ afirmaram fazer uso sempre.

A reinserção de um mesmo cateter periférico antes de acionar a proteção da agulha, em mais de uma punção, no mesmo paciente foi citada como prática rotineira por $48(26,7 \%)$ participantes; outros sete $(3,9 \%)$ responderam que realizavam esta prática algumas vezes; a maioria $(69,4 \%)$ sempre utilizava um novo cateter a cada punção. Não houve diferença significativa $(\mathrm{p}=0,10)$ ao comparar a categoria profissional e o uso de luvas, bem como ao comparar o reuso com acidente percutâneo notificados no setor de saúde e segurança do trabalhador $(\mathrm{p}=0,82)$. Já em relação ao reuso do cateter e a categoria profissional, houve diferença significativa $(\mathrm{p}=0,006)$, o percentual de Reuso $=1$ é significativamente superior no grupo de técnicos e auxiliares de enfermagem $(73,2 \%>48,1 \%)$; o percentual de Reuso $=2$ é significativamente superior no grupo Enfermeiro $(51,9 \%>22,2 \%)$. 


\section{Discussão}

Nesta pesquisa houve maior participação de técnicos e auxiliares de enfermagem. Isso pode ser justificado pela equipe de enfermagem ser composta $80 \%$ de técnicos e auxiliares e $20 \%$ enfermeiros. ${ }^{16}$

Quanto ao sexo, o masculino foi mais presente, resultado distinto de outros estudos encontrados na literatura nacional e internacional, ${ }^{7,17}$ bem como pesquisa realizada pelo Conselho Federal de Enfermagem (COFEN) em parceria com a Fundação Oswaldo Cruz, que embora revele uma tendência de masculização da profissão, 84,6\% dos trabalhadores desta área ainda são constituídos por mulheres. ${ }^{18}$ Em relação à faixa etária, a maioria possui idade entre 3039 anos, corroborando com outro estudo da literatura. ${ }^{19}$

Quanto à realização de Curso/Treinamento para uso de DSCE, apesar da sua obrigatoriedade, os dados desta investigação apontaram que a maioria dos trabalhadores da equipe de enfermagem respondeu não ter recebido nenhum treinamento, seja pelo fabricante ou pelo próprio hospital. O teste estatístico mostrou que os enfermeiros realizam mais treinamentos do que os técnicos e auxiliares de enfermagem.

A falta de motivação, incentivo e pouco investimento por parte dos gestores e a não liberação da chefia por causa dos treinamentos acontecerem no mesmo horário de trabalho, são algumas dificuldades para a não adesão dos profissionais de enfermagem às capacitações. ${ }^{20}$ Treinamentos para o uso correto de DSCE associados a programas de capacitação ou treinamento podem contribuír para a redução de acidentes com agulha e devem ser incentivados e realizados periodicamente. ${ }^{21}$

Os profissionais de enfermagem devem receber educação permanente, pois as tecnologias relacionadas à saúde sofrem constantes transformações, e atualização para acompanhar os avanços na área são necessárias. ${ }^{22}$ 
No Brasil, a Norma Regulamentadora № 32 estabelece que a capacitação da correta utilização do DSCE deve ser disponibilizada para os trabalhadores dos serviços de saúde pelas empresas que produzem ou comercializam materiais perfurocortantes. ${ }^{1,13}$

Antes da introdução de qualquer DSCE na área da saúde, é necessário avaliar a saúde e a segurança do trabalhador, a satisfação do usuário, a segurança e o conforto do paciente, para assim assegurar a adequação desse dispositivo na prática segura e eficaz. ${ }^{10}$ Os DSCE usados para a PVP podem apresentar características específicas segundo o modelo e fabricantes, como acionamento do mecanismo de proteção da agulha por técnica passiva ou ativa ou sistemas que impedem refluxo do sangue, reduzindo as chances de respingo ou contato com o sangue após a retirada da agulha e ou conexão do equipo. ${ }^{10-12}$ Neste sentido, identificar tais características e todos os requisitos necessários para o bom funcionamento do mesmo pode ser difícil para o profissional, uma vez que está em contato direto com uma gama de produtos usados na assistência hospitalar.

Quanto ao conhecimento dos profissionais de enfermagem em relação ao DSCE, identificou-se que a maioria soube responder informações quanto às suas características, como proteção da ponta da agulha ou contato com o sangue. Tais fatores são importantes para identificar o tipo de dispositivo usado e as características de segurança que oferecem ao profissional.

Tal resultado mostra a importânica da participação dos profissionais na escolha do produto que irão usar, bem como no envolvimento dos gestores. Autores de pesquisas relacionadas à satisfação e avaliação de DSCE apontaram ser fundamental a capacitação dos profissionais antes, durante e após um novo DSCE, sendo indispensável para evitar não só as exposições, como também gastos desnecessários à instituição. ${ }^{23-25}$

Nesta investigação, as luvas foram citadas pela maioria dos profissionais, mas por se tratar de um instrumento auto respondido, os dados podem estar superestimados. Neste 
aspecto, um estudo sobre os cuidados realizados pelos profissionais de enfermagem durante a PVP evidenciou que apesar da maioria responder que sempre usava luvas de procedimentos, um percentual ainda refere não usar e os motivos devem ser mais explorados, pois se trata de um procedimento de risco. ${ }^{2}$

\section{Conclusão}

Neste estudo identificou-se que a maioria dos profissionais de enfermagem mostrou conhecer as características do DSCE usado atualmente na instituição em que trabalham, embora relatassem não se sentirem protegidos com a utilização do cateter. Sobre as atitudes nos procedimentos realizados com o DSCE, a maior parte deles apresentou comportamento de segurança, o que não exclui o risco de exposição a material biológico e acidente com perfurocortante.

Portanto, ressalta-se a necessidade de que com a inserção de novos dispositivos, hospitais e fornecedores possam proporcionar aos profissionais de enfermagem programas de treinamentos, antes, durante e após a introdução de um novo produto, como os DSCE, além de medidas de prevenção à exposição com material biológico.

Os dados foram coletados em um único momento e em uma única instituição e, portanto, não refletem a realidade de outros cenários. Novos estudos com outras metodologias que permita a identificação e compreensão das atitudes de profissionais de enfermagem na PVP e DSCE serão importantes para a prática de enfermagem.

\section{Referências}

1. Brasil. Ministério do Trabalho e Emprego. Portaria n. 485, 11 de novembro de 2005. Aprova a Norma Regulamentadora n. 32 que dispõe sobre a segurança e saúde no trabalho em serviços de saúde. Diário Oficial da União, Brasília (DF); 2005 nov 16. Seção 1, p. 80-94. 
2. Melo EM, Aragão AL, Pessoa CMDP, Lima FET, Barbosa IV, Studart RMB. Cuidados dispensados pela equipe de enfermagem durante o procedimento de punção venosa periférica. Rev Enferm UFPE On Line [Internet]. 2015 mar [acesso em 2018 ago 04];9(3):1022-30. Disponível em: http://www.repositorio.ufc.br/bitstream/riufc/10892/1/2015_art_ivbarbosa.pdf

3. Vieira DB, Lima RO, Santana EB, Miranda GL, Cerqueira EM. Acidentes de trabalho com exposição a material biológico: ocorrências em profissionais de saúde em um hospital geral. Rev Baiana Saúde Pública [Internet]. 2016 out-dez [acesso em 2019 jul 10];40(4):821-31. Disponível em: http://rbsp.sesab.ba.gov.br/index.php/rbsp/article/view/1598/2135

4. Dulon M, Lisiak B, Wendeler D, Nienhaus A. Causes of needlestick injuries in three healthcare settings: analysis of accident notifications registered six months after the implementation of EU Directive 2010/32/EU in Germany. J Hosp Infect [Internet]. 2017 mar [acesso em 2018 ago 03];95(3):306-11. Disponível em: https://doi.org/10.1016/j.jhin.2016.11.015

5. Dornelles C, Carvalho LA, Thofehrn MB, Nunes NJDS, Fernandes HN. Exposição de profissionais de saúde ao material biológico: estudo no ambiente hospitalar. J Nurs Health [Internet]. 2016 [acesso em 2019 jul 15];1(1):64-75. Disponível em: https://periodicos.ufpel.edu.br/ojs2/index.php/enfermagem/article/view/5463/5329

6. Kasatpibal N, Whitney JD, Katechanok S, Ngamsakulrat S, Malairungsakul B, Sirikulsathean P, et al. Prevalence and risk factors of needlestick injuries, sharps injuries, and blood and body fluid exposures among operating room nurses in Thailand. Am J Infect Control [Internet]. 2016 jan [acesso em 2018 ago 1];44(1):85-90. Disponível em: https://doi.org/10.1016/j.ajic.2015.07.028

7. Lo W-Y, Chiou S-T, Huang N, Chien L-Y. Long work hours and chronic insomnia are associated with needlestick and sharps injuries among hospital nurses in Taiwan: a national survey. Int J Nurs Stud [Internet]. $2016 \mathrm{dez}$ [acesso em 2018 ago 01];(64):130-6. Disponível em: https://doi.org/10.1016/j.ijnurstu.2016.10.007

8. Menezes JA, Bandeira CS, Quintana M, Lima e Silva JCA, Calvet GA, Brasil P. Impact of a single safety-engineered device on the occurrence of percutaneous injuries in a general hospital in Brazil. Am J Infect Control [Internet]. 2016 fev [acesso em 2018 jun 02];42:174-7. Disponível em: https://doi.org/10.1016/j.ajic.2013.07.017

9. De Carli G, Abiteboul D, Puro V. The importance of implementing safe sharps practices in the laboratory setting in Europe. Biochem Med [Internet]. 2014 fev [acesso em 2019 jul 11];24(1):45-56. Disponível em: https://www.ncbi.nlm.nih.gov/pmc/articles/PMC3936965/

10. Adams D, Elliott TSJ. Safety-engineered needle devices: evaluation prior to introduction is essential. J Hosp Infect [Internet]. 2011 out [acesso em 2018 jun 02];79(2):174-5. Disponível em: https://doi.org/10.1016/j.jhin.2011.05.020 
Conhecimento e atitudes da equipe de enfermagem sobre cateter com dispositivo seguro... $\mid 14$

11. Seiberlich LE, Keay V, Kallos S, Junghans T, Lang E, McRae AD. Clinical performance of a new blood control peripheral intravenous catheter: a prospective, randomized, controlled study. Int Emerg Nurs [Internet]. 2016 mar [acesso em 2018 ago 01];(25):59-64. Disponível em: https://doi.org/10.1016/j.ienj.2015.08.005

12. Phillips EK, Conaway M, Parker G, Perry J, Jagger J. Issues in understanding the impact of the needlestick safety and prevention act on hospital sharps injuries. Infect Control Hosp Epidemiol [Internet]. 2013 set [acesso em 2018 ago 04];34(9):935-9. Disponível em: https://doi.org/10.1086/671733

13. Brasil. Ministério do Trabalho e do Emprego. Portaria n. 939, de novembro de 2008. Diário Oficial da União, Brasília (DF); 2008 nov 11. Seção 1, p. 238.

14. Fukuda H, Yamanaka N. Reducing needlestick injuries through safety-engineered devices: results of a Japanese multi-centre study. J Hosp Infect [Internet]. 2016 jan-mar [acesso em 2018 ago 01];92(2):147-53. Disponível em: https://doi.org/10.1016/j.jhin.2015.09.019

15. Beynon A. A quality improvement initiative to reduce needlestick injuries. Nurs Stand [Internet]. [acesso em 2019 jul 10];29(22):37-42. Disponível em: https://www.ncbi.nlm.nih.gov/pubmed/25627533

16. Fundação Oswaldo Cruz (Fiocruz); Conselho Regional de Enfermagem (COFEN). Pesquisa inédita traça perfil da enfermagem. [Internet]. 2015 [acesso em 2018 ago 01]. Disponível em: http://www.cofen.gov.br/pesquisa-inedita-traca-perfil-da-enfermagem_31258.html

17. Ferreira LA, Peixoto CA, Paiva L, Silva QCG, Rezende MP, Barbosa MH. Adesão às precauções padrão em um hospital de ensino. Rev Bras Enferm [Internet]. 2017 fev [acesso em 2018 jan 26];70(1):96103. Disponível em: http://dx.doi.org/10.1590/0034-7167-2016-0138

18. Conselho Federal de Enfermagem (COFEN). Pesquisa inédita traça perfil da enfermagem no Brasil [Internet]. 2015 [acesso em 10 jul 2019]. Disponível: http://www.cofen.gov.br/pesquisa-inedita-traca-perfildaenfermagem_31258.html

19. Silva SMS, Gomes IVMPS, Anjos MS. Acidente perfurocortante: conhecimento e uso de dispositivos de segurança. Revista Saúde.com [Internet]. 2016 jul [acesso em 2018 jan 20];12(2):522-7. Disponível em: http://www.uesb.br/revista/rsc/ojs/index.php/rsc/article/view/353

20. Macedo WTP, Figueiredo BM, Reis DSTD, Barros SHP, Ramos MCA, Silva SED. Adesão dos profissionais de enfermagem às práticas educacionais. Rev Pesqui Cuid Fundam [Internet]. 2019 [acesso em 2019 jul 18];11(4):1058-64. Disponível em:

http://www.seer.unirio.br/index.php/cuidadofundamental/article/view/6923/pdf_1

21. Darouiche HM, Chaabouni T, Hammami KJ, Akrout FM, Abdennadher M, Hammami A, et al. Occupational blood exposure among health care personnel and hospital trainees.

Int J Occup Environ Med [Internet]. 2014 jan [acesso em 2018 jan 02];5(1):57-61. Disponível em: http://www.theijoem.com/ijoem/index.php/ijoem/article/view/321/440 
22. Carvalho DC, Rocha JC, Gimenes MCA, Santos EC, Valim MD. Acidentes de trabalho com material biológico na equipe de enfermagem de um hospital do Centro-Oeste brasileiro. Esc Anna Nery Rev Enferm [Internet]. 2018 [acesso em 2018 jul 03];22(1): 01-8. Disponível em: http://www.scielo.br/pdf/ean/v22n1/pt_1414-8145-ean-2177-9465-EAN-2017-0140.pdf

23. Seiberlich LE, Keay V, Kallos S, Junghans T, Lang E, McRae AD. Clinical performance of a new blood control peripheral intravenous catheter: a prospective, randomized, controlled study. Int Emerg Nurs [Internet]. 2016 mar [acesso em 2018 jul 03];25:59-64. Disponível em: https://doi.org/10.1016/j.ienj.2015.08.005

24. Jagger J, Perry J. Safety-engineered devices in 2012: the critical role of healthcare workers in device selection. Infect Control Hosp Epidemiol [Internet]. 2013 jun [acesso em 2018 ago 04];34(6):615-8. Disponível em: http://dx.doi.org/10.12957/reuerj.2016.11518

25. Kevitt F, Hayes B. Sharps injuries in a teaching hospital: changes over a decade. Occup Med [Internet] 2015 mar [acesso em 2018 jul 03];64(8):01-4. Disponível em: https://doi.org/10.1093/occmed/kqu182

\section{Autor correspondente}

Nome: Priscila do Carmo Freitas de Carvalho

E-mail: prisciladcf@yahoo.com.br

Endereço: Rua Castelo de Óbidos, 201, bairro Castelo, Belo Horizonte, MG

CEP: $31330-320$

\section{Contribuições de Autoria}

\section{1 - Priscila do Carmo Freitas de Carvalho}

Concepção e planejamento do projeto de pesquisa, obtenção, análise e interpretação dos dados, redação e revisão crítica do artigo.

\section{2 - Gabriela da Cunha Januário}

Análise e interpretação dos dados, redação e revisão crítica do artigo.

\section{3 - Damiana Aparecida Trindade Monteiro}

Análise e interpretação dos dados, redação e revisão crítica do artigo

\section{4 - Silmara Elaine Malaguti Toffano}

Concepção e planejamento do projeto de pesquisa, obtenção, análise e interpretação dos dados, redação e revisão crítica do artigo. 
Conhecimento e atitudes da equipe de enfermagem sobre cateter com dispositivo seguro... $\mid 16$

\section{Como citar este artigo}

Carvalho PCF, Januário GC, Monteiro DAT, Toffano SEM. Conhecimento e atitudes da equipe de enfermagem sobre cateter com dispositivo seguro em um hospital brasileiro. Rev. Enferm. UFSM. 2019 [Acesso em: Anos Mês Dia];vol9 e50: P1-P17. DOI:https://doi.org/10.5902/2179769234767 